\title{
Quercetin ameliorates hypobaric hypoxia-induced memory impairment through mitochondrial and neuron function adaptation via the PGC-1 $\alpha$ pathway
}

\author{
Peng Liu, Dan Zou ${ }^{1}$, Long Yi, Mingliang Chen, Yanxiang Gao, Rui Zhou, Qianyong Zhang, \\ Yong Zhou, Jundong Zhu, Ka Chen* and Mantian Mi* \\ Research Center for Nutrition and Food Safety, Institute of Military Preventive Medicine, Third Military Medical \\ University, Chongqing Key Laboratory of Nutrition and Food Safety, Chongqing Medical Nutrition Research Center, \\ Chongqing, PR China
}

\begin{abstract}
.
Purpose: Acute hypobaric hypoxia $(\mathrm{HH})$ causes persistent cognitive impairment, affecting memory function specifically. Mitochondrial dysfunction and synaptic morphological change were the prominent pathological features of HH exposure on brain. Quercetin, a flavonoid found in fruits, vegetables, leaves and grains, is reported to prevent ischemia induced by neuronal injury. This study investigated the efficacy of quercetin to ameliorate HH-induced memory deficit.

Methods: Rats were exposed to HH equivalent to $5000 \mathrm{~m}$ for 7 days in a decompression chamber and received quercetin daily $(50,75$ or $100 \mathrm{mg} / \mathrm{kg} \cdot \mathrm{bw})$ via gavage during the period of exposure. Cognitive performance was assessed by the Morris water maze test. In vitro,the effect of quercetin was tested in hippocampus tissue.

Results: Quercetin, especially at $100 \mathrm{mg} / \mathrm{kg} \cdot \mathrm{bw}$, significantly reduced HH-induced memory decline. Meanwhile, HH-induced hippocampus mitochondrial and synaptic lesions were ameliorated by quercetin. Furthermore, quercetin regulated the expression of sirtuin 1(Sirt1), PGC-1 $\alpha$, and the proteins related with mitochondrial biogenesis and dynamics. Moreover, quercetin increased expression of fibronectin type III domain-containing protein 5 (FNDC5) and brain-derived neurotrophic factor (BDNF), showing the PGC-1 $\alpha /$ FNDC5/BNDF pathways might be involved in neuronal adaptation.

Conclusions: The results suggest quercetin has prophylactic potential for amelioration of HH-induced memory impairment, which is associated with the mitochondrial and neuronal adaptation in hippocampus.

Keywords: Hypobaric hypoxia, memory impairment, mitochondria, neuroprotection, PGC-1 $\alpha$, quercetin

Abbreviation

HH hypobaric hypoxia

Sirt1 sirtuin 1

\footnotetext{
${ }^{1}$ Peng Liu and Dan Zou contribute evenly in this paper.

*Corresponding author: Mantian Mi and Ka Chen, Research Center for Nutrition and Food Safety, Institute of Military Preventive Medicine, Third Military Medical University, Chongqing Key Laboratory of Nutrition and Food Safety, Chongqing Medical Nutrition Research Center, Chongqing 400038, PR China. Tel.: +86 013508 305561; Fax: +86 236875 2305; E-mail: mimantian@outlook.com; and Tel.: +86 015111985982; Fax: +86 236875 2305; E-mail: chen_ka@sina.com (Ka Chen).
}

FNDC5 fibronectin type III domain-containing protein 5

BDNF brain-derived neurotrophic factor

PGC- $1 \alpha$ peroxisome proliferator-activated receptor- $\gamma$ coactivator- $1 \alpha$

NOR normoxia

HYP hypoxia

ROS roseroot

QUE quercetin

MWM Morris water maze

ND1 NADH-CoQoxidoreductase 1

Nrf1 nuclear respiratory factor 1
\end{abstract}


Tfam mitochondrial transcription factor A

Mfn1 Mitofusin 1

Mfn2 Mitofusin 2

Drp1 Dynamin-related protein 1

Fis1 fission 1 homolog

Vdac1 Voltage-dependent anion-selective channel protein 1

TEM Transmission electron microscope

\section{Introduction}

Hypobaric hypoxia $(\mathrm{HH})$, often present at high altitudes (mountains, airplanes, space shuttles etc.), is characterized by low barometric pressure with a consequent fall in the partial pressure of oxygen. $\mathrm{HH}$ is known to have deleterious effects on brain functions, leading to impairment of perception, memory, judgment and attention (Kramer et al., 1993; Shukitt-Hale et al., 1998). The hippocampus, a part of the brain involved in learning and memory, is prone to impairment by $\mathrm{HH}$ (Morris et al., 1982). HH-induced cognitive deficit has attracted special concern because this problem compromises mental performance. Moreover, cognitive decline at high altitude is associated with sustained neuronal damage and persists for a long time even after returning to sea level (Cavaletti et al., 1990; Cavaletti and Tredici 1993; Shukitt-Hale et al., 1996). Therefore, it is important to provide protective measures against acute $\mathrm{HH}$ exposure, especially for populations at risk, including military personnel and rescue workers.

Quercetin $\left(3,3^{\prime}, 4^{\prime}, 5,7\right.$-pentahydroxyflavone $)$ is a flavonoid compound in fruits, vegetables, leaves and grains (Silva et al., 2008). Besides its well-known antioxidant property, the involvement of quercetin in the activation of the key inducible metabolic regulator, peroxisome proliferator-activated receptor- $\gamma$ coactivator-1 $\alpha$ (PGC-1 $\alpha)$, has been shown in recent years (Davis et al., 2009). PGC- $1 \alpha$ is a co-activator controlling mitochondrial biogenesis and respiration to meet the energy demands of a changing environment (Aquilano et al., 2010). Recent studies showed PGC- $1 \alpha$ was involved in adaptation of neuron function through regulating brain-derived neurotrophic factor (BDNF) expression via fibronectin type III domain-containing protein 5(FNDC5) (Wrann et al.,
2013). Mitochondrial dysfunction and altered synaptic plasticity were the prominent pathological features of $\mathrm{HH}$ on brain (Magalhaes et al., 2005; Maiti et al., 2008); therefore, it is plausible to speculate that PGC- $1 \alpha$ activation will reveal potential therapeutic targets for attenuation of memory dysfunction after exposure to $\mathrm{HH}$. The effectiveness of quercetin has been established in spatial memory improvement and neuronal injury amelioration under condition of ischemia (Pu et al., 2004). Moreover, it is of interest to explore whether the neuroprotective effects of quercetin are related to activation of the PGC- $1 \alpha$ pathway.

This study was designed to evaluate the effects of quercetin supplementation on memory function of rats exposed to $\mathrm{HH}$ and to observe alterations of mitochondrial biogenesis, dynamics and respiratory chain activity and the plasticity of synapses in the hippocampus. In order to investigate the underlying mechanism, we asked whether the Sirt1/PGC-1 $\alpha / \mathrm{Nrf}-1 / \mathrm{Tfam}$ and PGC-1 $\alpha /$ FNDC5/BNDF pathways had key roles in mitochondrial and neuronal adaptation of rats exposed to $\mathrm{HH}$.

\section{Materials and methods}

\subsection{Animals}

Adult male Sprague Dawley ${ }^{\circledR}$ rats $(n=72)$ with an average body weight of $200 \pm 25 \mathrm{~g}$ were obtained from the Experimental Animal Center of the Third Military Medical University, Chongqing, PR China. Rats maintained in polypropylene cages $(50 \times 36 \times 20 \mathrm{~cm})$ with access to a pelleted diet and water ad libitum were exposed to $12 \mathrm{~h}$ light $/ 12 \mathrm{~h}$ dark cycles. The Ethical Committee of the Third Military Medical University, Chongqing, PR China approved all the experimental protocols for this study, and adequate measures were taken to minimize animal suffering and limit the number of animals used.

\subsection{Experimental design}

After trained in the Morris Water Maze for 7 days, the rats were divided randomly into six groups ( $n=12$ /group), followed by a probe test on the eighth day. Then, excepting for the Group NOR, all groups were exposed to $\mathrm{HH}$ for 7 days. During exposure to $\mathrm{HH}$ for 7 days, group HYP received no drug 
treatment; group ROS was treated with roseroot $(500 \mathrm{mg} / \mathrm{kg} \cdot \mathrm{bw})$, which is a plant extract used regularly to protect the body from high altitude sickness; all groups were exposed to $\mathrm{HH}$ forgroups QUE-50, QUE-75 and QUE-100 were exposed to $\mathrm{HH}$ and were treated with quercetin $(50,75$ and $100 \mathrm{mg} / \mathrm{kg}$ bw, respectively). Roseroot and quercetin were dissolved in distilled water and administered daily by gavage at 9:00 am during exposure to $\mathrm{HH}$ for 7 days. After 7 days' hypoxic exposure, memory function was assessed by a probe test in the Morris water maze. Subsequently, rats were anesthetized and perfusion fixed with $4 \%$ poly-formaldehyde or sacrificed by cervical dislocation, and then the hippocampus were dissected out in ice-cold condition and stored at $-80^{\circ} \mathrm{C}$ until use.

\subsection{Exposure to $\mathrm{HH}$}

Exposure to a simulated altitude of $5000 \mathrm{~m}$ was achieved in an altitude chamber with an atmospheric pressure of $405.35 \mathrm{mmHg}$ and $10.9 \%$ oxygenat a temperature of $23-25^{\circ} \mathrm{C}$ and relative humidity of 55-60\%. Except for the NOR group, all groups were subjected to a $\mathrm{HH}$ chamber. The partial pressure of nitrogen decreased as the total pressure decreased on ascent; the nitrogen content did not change. The ascent to altitude took $20 \mathrm{~min}$ at a constant rate of $300 \mathrm{~m} / \mathrm{min}$. The atmospheric pressure in the chamber was brought to the equivalent to sea level every day for $30 \mathrm{~min}$ to replenish food and water for administration of drugs as appropriate.

\subsection{Morris water maze}

Memory consolidation and retention was evaluated using the Morris water maze (MWM) system as described (Morris et al., 1982; Morris, 1984). Rats were required to learn the location of a hidden platform in a circular pool (diameter $150 \mathrm{~cm}$, height $60 \mathrm{~cm}$ black background) filled with water at $23.5^{\circ} \mathrm{C}$. A circular black platform (diameter $12 \mathrm{~cm}$, height $23 \mathrm{~cm}$ ) was placed $1.5 \mathrm{~cm}$ below the surface of the water (submerged) in the center of the target quadrant. An overhead camera and a Videomex computer-assisted tracking system recorded the position of the rat in the maze, which enables the latency (time taken to reach the platform) to be measured in seconds, path length (distance traveled by the rat to find the platform) in centimeters and other parameters, including entry point, time and path length in each quadrant. The water tank was divided into imaginary quadrants southeast, northeast, northwest and southwest. The platform was located in the center of the target quadrant (southeast in this study) throughout the experiment.

Training. All trials in each experiment were done at the same time of day (09:00-11:00 am). The animals were divided into different groups and trained for 7 th days and a probe test was performed on the 8th day. Two days before the training, each rat underwent training, during which it was required to swim for $60 \mathrm{~s}$ in the pool without a platform. Each rat underwent four trials per day with an interval of 5 min between trials. Each trial consisted of placing the rat gently by hand into the water facing the wall of the pool at a different starting point in each trial and allowing $60 \mathrm{~s}$ to locate the submerged platform. After finding the platform, the rat was allowed to remain there for $30 \mathrm{~s}$, then held gently and dried for another $30 \mathrm{~s}$ until the next training swim and placed back into its home cage after the training period. Any rat failing to locate the platform within $60 \mathrm{~s}$ was guided to the platform by hand. During the inter-training delay, the rat was kept warm by warm air from a hair drier.

Probe Test. Twenty four hours after the last day of training, as well as after exposure to $\mathrm{HH}$ for 7 days, the rats were given a $1 \mathrm{~min}$ probe test, which was designed to assess the memory consolidation and retention. It consisted of a single trial in which the rats were allowed to swim for $60 \mathrm{~s}$, with the platform removed from its position.Here, escape latencies, path length and swim paths were recorded with a Videomex tracking system for assessing memory consolidation and retention.

\subsection{Transmission electron microscope}

The hippocampus were fixed for $18-20 \mathrm{~h}$ in the $2.5 \%$ $(\mathrm{v} / \mathrm{v})$ glutaraldehyde, and then in $1 \%(\mathrm{w} / \mathrm{v}) \mathrm{OsO}_{4}$ for $1 \mathrm{~h}$. The fixed slices were dehydrated in an ascending series of ethanol finishing with absolute alcohol and embedded in EPON resin. Ultra-thin sections $(70 \mathrm{~nm}$ thick) cut from the tissue slices were stained with uranyl acetate and lead citrate and examined in a JEM1400 electron microscope (Jeol, Japan). Photographs of the sections were taken in a JEM-100CX electron microscope at magnifications of $17,500 \times$. For each hippocampus, five random sections were examined and, from each section, six random pictures were taken. The area of mitochondrial surface and postsynaptic density was estimated using the point counting 
method (Weibel, 1979). The length of mitochondria was measured using COREL Photo Paint on micrographs. Meanwhile, the number of mitochondria was also counted on micrographs. Counting was performed using blind method. These values were used to calculate the average surface area, length and number of mitochondria for each group.

\subsection{Detection of the $m t D N A$}

Total DNA was extracted from individual hippocampus tissue samples using a DNA extraction kit according to the manufacturers' instructions (Genmed Scientifics Inc. USA). The levels of mitochondrial ND1 (NADH-CoQoxidoreductase 1) relative to standard $\beta$-actin, which is the relative number of mtDNA copies, were determined by quantitative PCR using the SYBR Green mixtures and specific primers.ND1: forward 5'-GAA CCC ATA CGC CCC CTA AC-3' reverse $5^{\prime}$-GCT CGT AGG GCT CCG AAT AG- $3^{\prime} \beta$ actin: forward $5^{\prime}$-CCACCATGTACCCAGGCATT- ${ }^{\prime}$ reverse 5'-CGGACTCATCGTACTCCTGC-3'.

\subsection{Measurement of ATP levels, mitochondrial complex I, II, IV and V activity}

ATP levels were measured using the ATP Assay Kit (Beyotime, China) according to manufacturer's protocol. The activity of complex I, II, IV and V in hippocampus homogenates $(50 \mu \mathrm{g}$ of total protein) was measured with a MitoProfile Rapid Micro-plate assay kit according to the manufacturer's instructions (catalog nos. ab109721, ab109908 and ab109911, respectively, Abcam, USA). Complexes I, II, IV and V were immune-captured within the wells of the microplates and enzyme activity was measured by a kinetic colorimetric assay kit (Thermo Fisher Scientific Inc.) as described by the manufacturer. Total protein in hippocampus homogenates was estimated using a protein assay kit (DC, Bio-Rad, USA).

\subsection{Western blotting}

After SDS-PAGE (12\% polyacrylamide gel), total protein, nuclear proteins or mitochondrial proteins for immunoblotting analysis were transferred electrophoretic to a nitrocellulose membrane (BioRad) at $220 \mathrm{~mA}$ for $90 \mathrm{~min}$. Once the transfer was complete, membranes were blocked with $5 \%(\mathrm{v} / \mathrm{v})$ skimmed milk for $2 \mathrm{~h}$ and immunoblotted overnight at $4{ }^{\circ} \mathrm{C}$ with the following antibodies: $\beta$-actin (ZSGB-BIO), Vdac1(Abcam), Lamin B (Abcam), PGC-1 $\alpha$ (Abcam), Sirt1 (Cell Signaling Technology), nuclear respiratory factor 1 (Nrf1) (R\&D Systems), mitochondrial transcription factor A (Tfam) (Abcam), Mitofusin 1 (Mfn1) (Abcam), Mitofusin2 (Mfn2) (Cell Signaling Technology), Dynamin-related protein 1 (Drp1) (Cell Signaling Technology), fission 1 homolog (Fis1) (Abcam), FNDC5 (Abcam) and BDNF (Abcam). Blots were incubated with anti-rabbit/mouse/goat secondary antibody horseradish peroxidase-conjugated and visualized by the ECL chemiluminescence method (Millipore Corporation, Billerica, MA, USA). The monoclonal anti- $\beta$-actin antibody, anti-Lamin B antibody and anti-Vdac1 were used at a dilution of 1:5000 as a control for protein loading normalization in gels.

Levels of protein expression were determined by densitometry of the bands using Quantity One software (Bio-Rad Lab., Hercules, CA, USA). Fold changes were quantified as the target protein equal to the corresponding internal control (Lamin B, Vdac1 or $\beta$-actin) in the groups.

\subsection{Statistical analysis}

Quantitative data were expressed as mean \pm S.E. and analyzed by one-way ANOVA. Multiple comparisons between the groups were done with Tukey's post $h o c$ tests. The level of statistically significant difference was set at $p<0.05$ for all statistical analysis.

\section{Results}

\subsection{Quercetin protects the memory ability of rats}

HH can impair the spatial learning and memory ability of rats (Muthuraju et al., 2009). We thus sought to determine whether quercetin improved $\mathrm{HH}$-induced memory performance deficits. Before exposure to $\mathrm{HH}$, the rats were trained for 7 days to find a hidden platform using spatial cues located throughout the room. All groups showed a reduced latency and path length needed to find the hidden platform across training days. All groups performed similarly, and the escape latency and path length did not differ significantly among groups (Fig. 1A and B). In the probe test phase that occurred $24 \mathrm{~h}$ after the last training day, rats were given a 1 min trial to measure the percentage of total time and the percentage of total path length they spent in the 
A

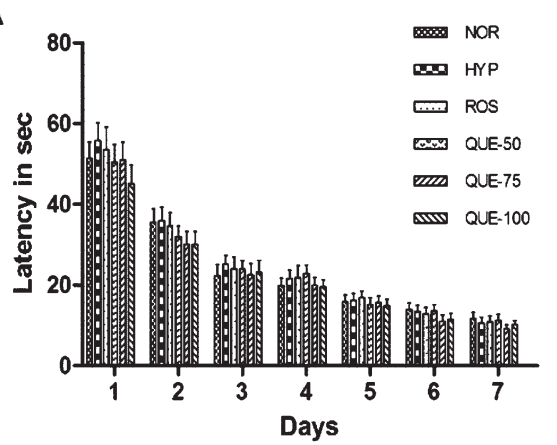

C

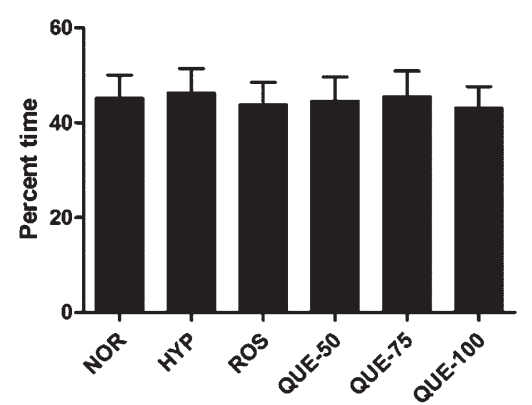

E

Probe test 2

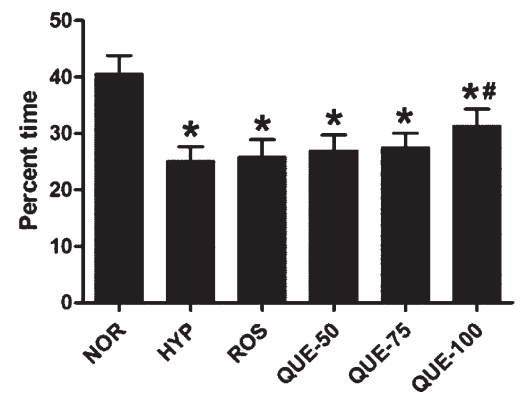

B

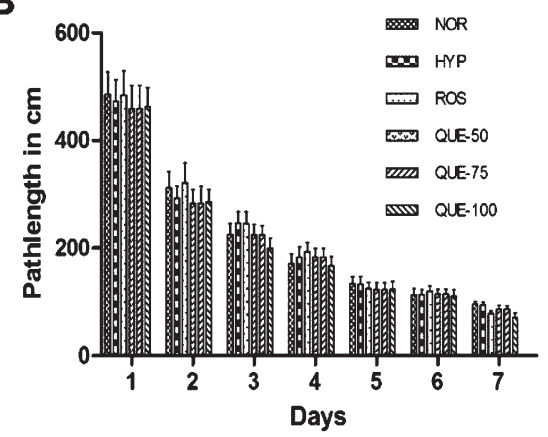

Probe test 1

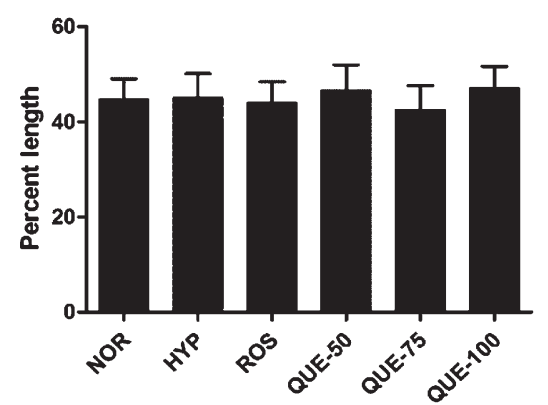

F

Probe test 2

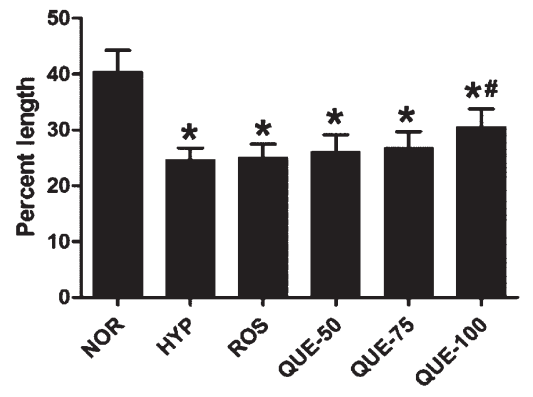

Fig. 1. Rats treated with quercetin after exposure to HH performed better in a Morris water maze. (A and B) From days 1-7, rats were trained with a hidden platform to test their spatial ability. We measured the latency and length needed to find the hidden platform. There was no significant difference among groups. (C and D) On day 8 , memory was evaluated with a probe test. The graphs represent the percentage of time (out of $60 \mathrm{~s}$ ) and path length that the rat took in the quadrant that contained the hidden platform (target quadrant). All groups took more time and greater distance in the target quadrant during the test. However, there was no significant difference among groups. (E and F) On day 15 , after exposure to HH for 7 days, we evaluated memory with a probe test. Group QUE-100 took significantly longer time and swam a greater distance compared to group HYP. ${ }^{*} p<0.05 v s$. group NOR and ${ }^{\#} p<0.05 v s$. group HYP. Data are expressed as mean \pm S.E.

target quadrant of the maze. There was no significant difference among groups (Fig. 1C and D). After exposure to $\mathrm{HH}$ for 7 days, all groups were also given a $1 \mathrm{~min}$ trial to assess memory ability. Group QUE-100 spent a significantly larger proportion of time $(31.36 \pm 2.97 \%$ of the time) and path length (30.56 $\pm 3.19 \%$ of the length) in the target quadrant (the quadrant in which the platform was located during the hidden platform training) compared to group HYP $(25.12 \pm 2.54 \%$ of the time and $24.72 \pm 2.04 \%$ of the length) (Fig. $1 \mathrm{E}$ and F).
The results for the proportion of time and length in the target quadrant of groups HYP and ROS were not significantly different.

\subsection{Quercetin induces the ultrastructural changes of mitochondria and synapse of hippocampus}

Transmission electron microscope (TEM) images of mitochondrial changes and synapses in rat hippocampus neurons after exposure to $\mathrm{HH}$ were 
A

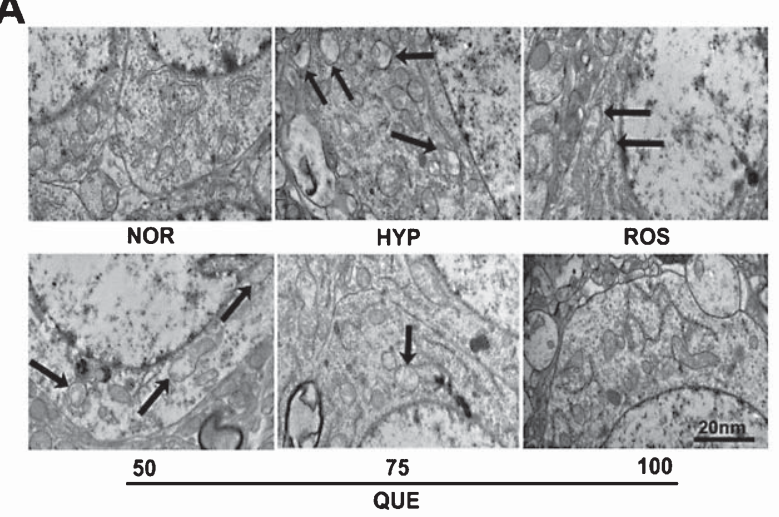

C

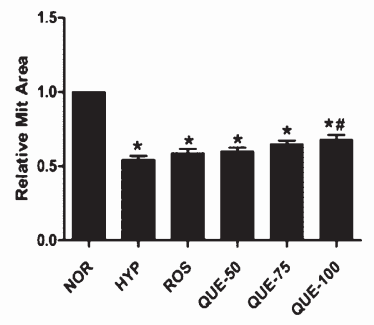

D

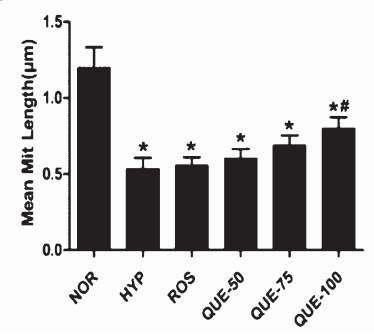

B
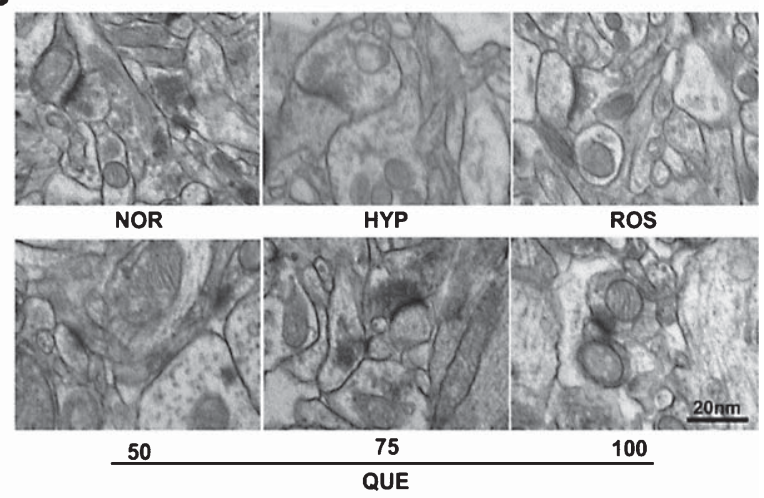

E

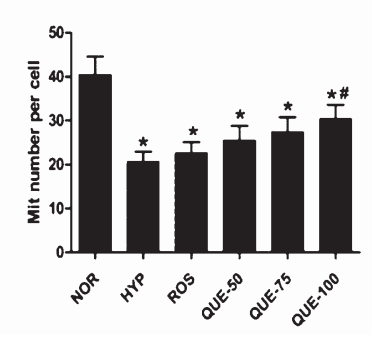

F

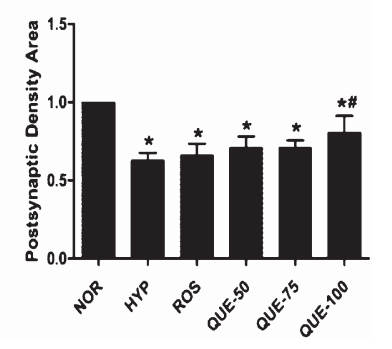

Fig. 2. Ultrastructural changes of neurons in the hippocampus under a TEM. (A) The changes of mitochondrial morphology in all groups. Magnification 17,500 $\times$. The presence of mitochondria with cristae loss (black arrows) are shown in micographs for each group. (B) The changes of synapsis structure in all groups. Magnification 17,500×. Quantitative analysis for all groups: (C) mitochondrial area, (D) mitochondrial length, (E) the number of mitochondria per cell, (F) postsynaptic density area. Data are expressed as mean \pm S.E. * $p<0.05 v s$. group NOR and ${ }^{\#} p<0.05$ vs. group HYP.

captured. Representative TEM images of all groups were showed in Fig. 2A. In group NOR, the mitochondria were mainly tubular and with normal structure. In group HYP, several mitochondria were large, swollen, showed a loss of internal cristae structure. In the QUE groups, especially QUE-100, there were several larger but morphologically intact mitochondria with normal structure. In groups QUE-50 and QUE75 , there were increased numbers of large, swollen mitochondria with rupture/loss of internal cristae, whereas some cells still had morphologically intact mitochondria. Morphometric analysis of mitochondria revealed clearly larger mitochondria in quercetintreated rats as compared to that of $\mathrm{HH}$-exposed rats. This amplification of the mitochondria was reflected both in the quantification of mitochondrial surface area and length (Fig. 2C and D). Consistent with enhanced mitochondrial biogenesis, a marked increase in the number of mitochondria per cell was also noted (Fig. 2E).

Figure 2B shows representative TEM images of synapses of hippocampus neurons in all groups. In group NOR, the postsynaptic density was thick and mitochondria of axons have normal structure with visible clear cristae. In group HYP, the postsynaptic density was thin and several mitochondria were swollen, showing a loss of internal cristae structure. In group ROS, the postsynaptic density was similar to group HYP. In the QUE groups, especially QUE100 , the postsynaptic density was thicker compared to group HYP. This difference of the postsynaptic density was shown in the quantification of surface area (Fig. 2F).

\subsection{Quercetin affects the amount of mtDNA in rats}

We measured the amount of mtDNA present after exposure to $\mathrm{HH}$. The levels of ND1 relative to standard $\beta$-actin were significantly lower in group HYP compared to group NOR. The levels in all QUE groups were significantly higher compared to group HYP (Fig. 3). 


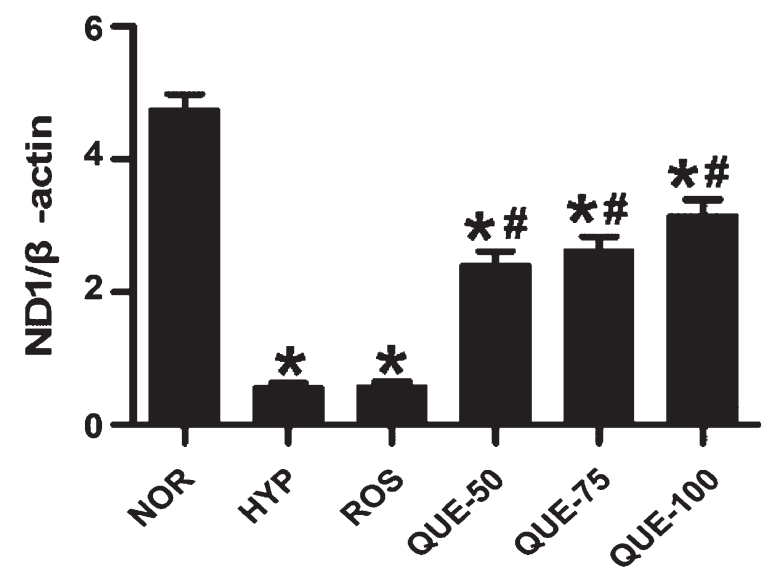

Fig. 3. Effect of quercetin on the mtDNA copy number in the hippocampus. The amount of mtDNA in the hippocampus of different groups of rats was determined by measuring the levels of ND1 relative to standard $\beta$-actin by quantitative PCR. Data are expressed as mean \pm S.E. ${ }^{*} p<0.05 v s$. group NOR and ${ }^{\#} p<0.05 v s$. group HYP.

\subsection{Quercetin protects against the reduction of Sirt1 and PGC-1 $\alpha$ expression}

Treatment with all doses (50, 75 and $100 \mathrm{mg} / \mathrm{kg} \cdot \mathrm{bw})$ of quercetin resulted in marked increased expression of Sirt 1 and PGC- $1 \alpha$ in the nuclear of hippocampus neurons compared to the group HYP (Fig. 4). We found the expression of Sirt1 and PGC- $1 \alpha$ had ascendant tendency with the dose raised. Also, the expression of Sirt 1 and PGC- $1 \alpha$ of the group ROS negligibly increased, but did not reach the expression levels of the groups QUE-50, QUE-75 and QUE-100 (Fig. 4).

\subsection{Quercetin protects against reduced enzyme activity of respiratory chain complexes}

Analysis of enzyme activity of respiratory chain complexes revealed significantly lower activity of complexes I, II, IV and V in group HYP compared to group NOR (Fig. 5A-D). The QUE groups showed significantly higher activity of complexes, and compared to group HYP (Fig. 5B, C and D). By contrast, there was no significant difference in the activity of complex I in any QUE group compared to group HYP (Fig. 5A). Figure 5E demonstrates that $\mathrm{HH}$ induced a significant decrease in hippocampal ATP levels, andquercetin treatment $(100 \mathrm{mg} / \mathrm{kg} \cdot \mathrm{bw})$ partly prevented $\mathrm{HH}$-induced decrease in ATP.

\subsection{Quercetin up-regulates mitochondrial biogenesis-related proteins}

The mtDNA relative numbers obtained by RT-PCR were significantly different in group QUE-100 compared to group HYP. We asked whether the PGC- $1 \alpha$ signaling pathway was a candidate for the regulation of mitochondrial biogenesis in vivo and whether treatment with quercetin influenced expression of the PGC- $1 \alpha$ signaling pathway proteins. Western blotting confirmed that quercetin $(100 \mathrm{mg} / \mathrm{kg} \cdot \mathrm{bw})$ increased the

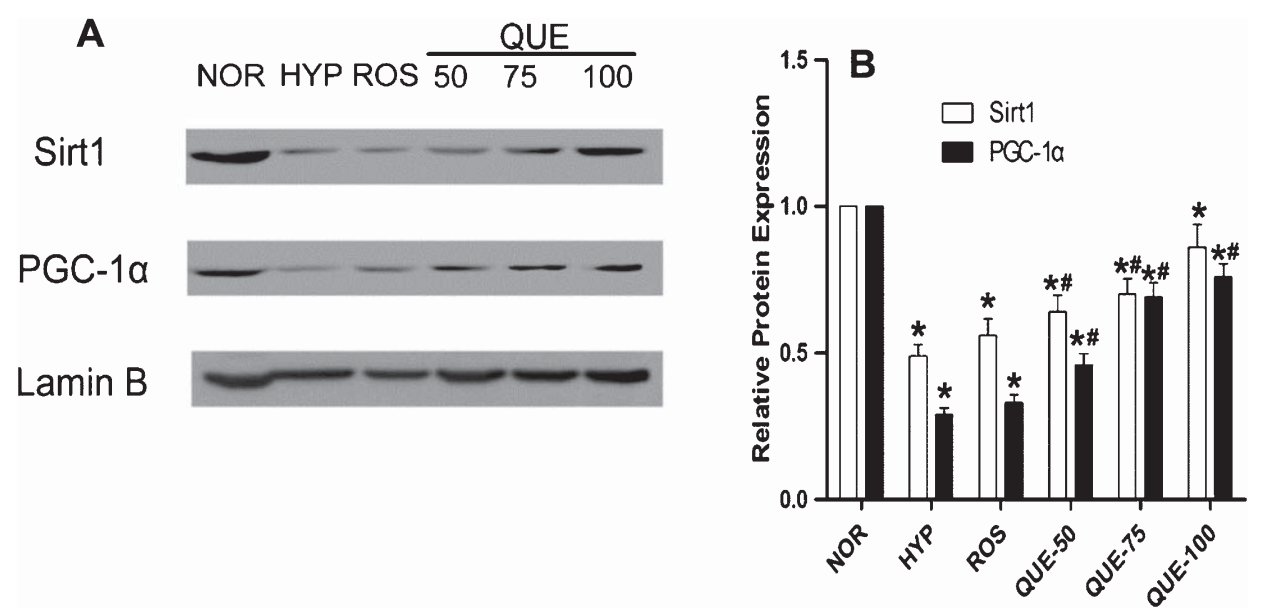

Fig. 4. Treatment with quercetin increased the expression of Sirt1 and PGC-1 $\alpha$ in the hippocampus. (A) Representative Western blot images. (B) Quantitative analysis showed treatment with quercetin increased protein levels of Sirt1 and PGC-1 $\alpha$ significantly compared to group HYP. ${ }^{*} p<0.05 v s$. group NOR and ${ }^{\#} p<0.05 v s$. group HYP. 
A Complex-I
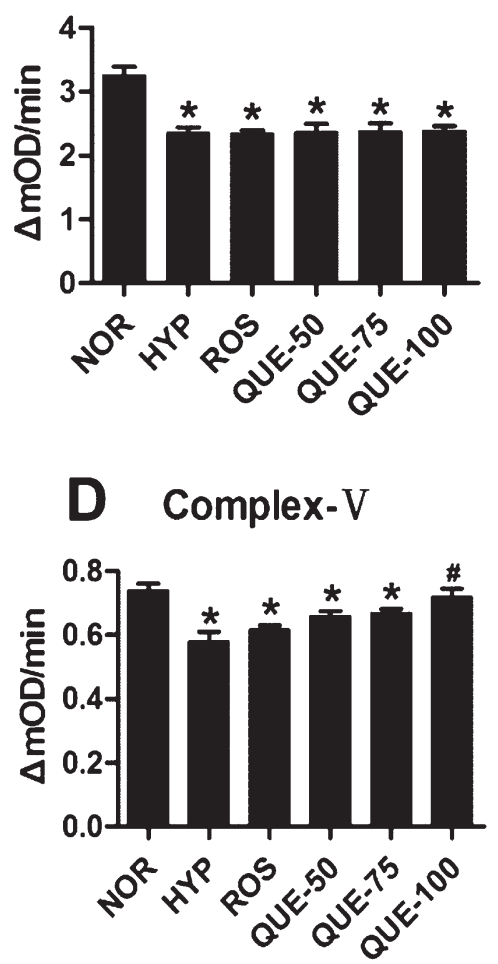

B Complex-II
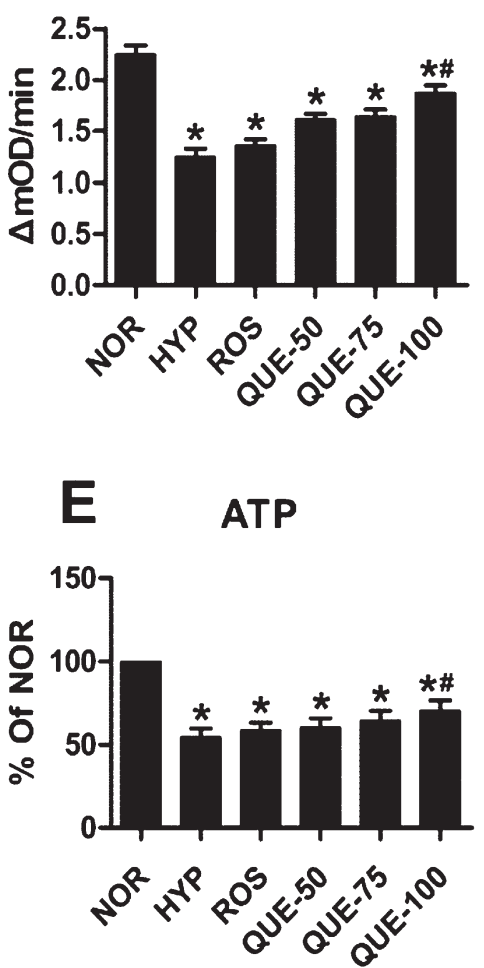

C Complex-IV

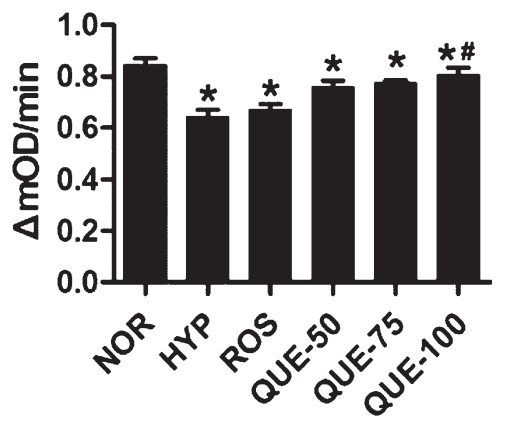

Fig. 5. Mitochondrial respiratory chain activity and ATP levels. Mitochondrial complex activities were measured in hippocampus tissue according to the manufacturer's instructions (catalog nos ab109721, ab109908 and ab109911, respectively, Abcam, USA): (A) complex I; (B) complex II; (C) complex IV; (D) complex V. (E) ATP levels were measured for all groups. Each bar represents mean \pm S.E. mOD, mitochondrial optical density. ${ }^{*} p<0.05 v s$. group NOR and ${ }^{\#} p<0.05$ vs. group HYP.

levels of Nrf1 and Tfam in homogenates of whole hippocampus significantly compared to the group HYP (Fig. 6A and B). Quantitative analysis indicated $\mathrm{HH}$ inhibited expression of Nrf1 and Tfam proteins (Fig. 6A and B). On the basis of these studies, we suggest that treatment with quercetin may cause the activation of PGC- $1 \alpha$ signaling in the hippocampus.

\subsection{Quercetin regulates mitochondrial fusion/fission-related proteins}

Mitochondrial number is related to the balance between mitochondrial fusion and fission events. As described, $\mathrm{HH}$ induced a reduction of the mean mitochondrial number, but the reduction was smaller when rats were treated with quercetin (Fig. 2E). By means of Western blotting, we investigated the expression of mitochondrial fusion/fission-related proteins
Drp1/Fis1 and Mfn1/Mfn2 in order to assess whether $\mathrm{HH}$ and treatment with quercetin were able to modulate mitochondrial dynamics. We used three concentrations of quercetin to provide an overview of mitochondrial response.

As expected, $\mathrm{HH}$ induced increased expression of mitochondrial fission-related proteins Drp1(Fig. 7A and B) and Fis 1 (Fig. 7C and D), suggesting increased numbers of fission events. Interestingly, the QUE groups had a significant decrease of fission-related proteins Drp1 and Fis1 compared to group HYP, suggesting quercetin down-regulated mitochondrial fission even under conditions of $\mathrm{HH}$. The expression of fusion-related proteins Mfn1 and Mfn2 was changed significantly by exposure to $\mathrm{HH}$ and by treatment with quercetin (Fig. 7C and D). These results allow us to suggest quercetin has a critical role in the modulation of mitochondrial dynamics. 

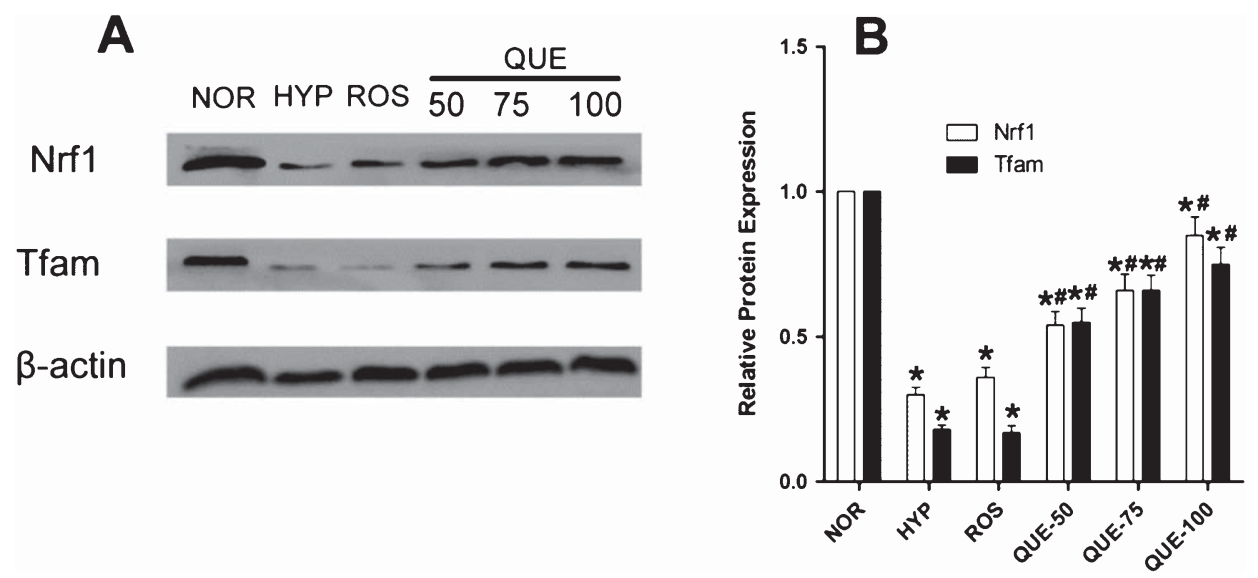

Fig. 6. Treatment with quercetin increased PGC-1 $\alpha$ signaling pathway components in the hippocampus. (A) Representative Western blot images. (B) Quantitative analysis showed that quercetin increased protein levels of Nrf1 and Tfam significantly compared to group HYP. ${ }^{*} p<0.05 v s$. group NOR and ${ }^{\#} p<0.05 v s$. group HYP.

\subsection{Quercetin regulates expression of FNDC5 and $B D N F$}

As mentioned above, BDNF is a major mediator of certain beneficial effects on the brain. Considering the induction of FNDC5 is a part of the transcriptional response to PGC- $1 \alpha$ in the hippocampus, we investigated the effects of quercetin on expression of FNDC5 and BDNF. Quercetin at the higher doses (75 and $100 \mathrm{mg} / \mathrm{kg} \cdot \mathrm{bw}$ ) significantly increased expression of FNDC5 and BDNF compared to the group HYP (Fig. 8A and B).

\section{Discussion}

Increasing attention has been paid to the effect of high-altitude exposure on mental performance. Earlier studies have shown that dexamethasone, acetazolamide, acetylcholinesterase inhibitors, corticosterone synthesis inhibitor and L-Type calcium channels antagonists could protect subjects from cognitive impairment induced by HH (Jobe et al., 1991; Wang et al., 2013). In fact, many travelers in high altitude prefer to take prophylactic dietary supplements as preventive measure for high altitude sickness. Flavonoids, a group of polyphenol compounds, are diverse in chemical structure and characteristics. They occur naturally in fruit, vegetables, nuts, seeds, flowers, and bark and are an integral part of the human diet (Das and Ratty, 1986; Hackett, 1986). They have been reported to exhibit a wide range of biological effects, including antibacterial, antiviral, anti-inflammatory, anti-allergic (Hanasaki et al., 1994), and vasodilatory (Duarte et al., 1993) actions. In this study, we used an $\mathrm{HH}$ paradigm to examine the efficacy of quercetin against $\mathrm{HH}$-induced memory impairment and to explore the possible role of PGC- $1 \alpha$-mediated signaling in quercetin-mediated neuroprotection. The results showed daily treatment with quercetin during 7 days of $\mathrm{HH}$ ameliorated $\mathrm{HH}$-induced memory impairment and prompted the role of Sirt1-mediated PGC- $1 \alpha$ activation, which led to up-regulation of the PGC$1 \alpha /$ Nrf-1/Tfam and PGC- $1 \alpha /$ FNDC5/BNDF pathways coupled with mitochondrial and neuronal adaptation to prevent neuron dysfunction.

\subsection{Quercetin ameliorates HH-induced memory deficit}

The reference and working memory of rats were impaired when exposed to HH (Muthuraju et al., 2009). In this study, the classical Morris Water Maze test was utilized to assess the spatial reference memory of rats. The results of the present study showed quercetin, especially at a dose of $100 \mathrm{mg} / \mathrm{kg} \cdot \mathrm{bw}$, significantly improved spatial reference memory in rats exposed to $\mathrm{HH}$, as evident from the significant increase in time and path-length spent in the target quadrant. In line with our findings, several studies documented the ameliorative memory-enhancing effect of the quercetin under several stress and diseased conditions (Kumar et al., 2008; Bhutada et al., 2010; Sriraksa et al., 2012). Moreover, Prasad et al. (2013) have also showed 
A

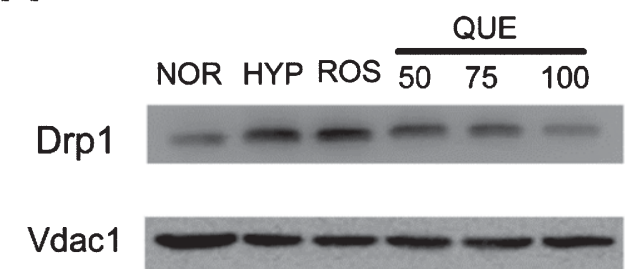

C

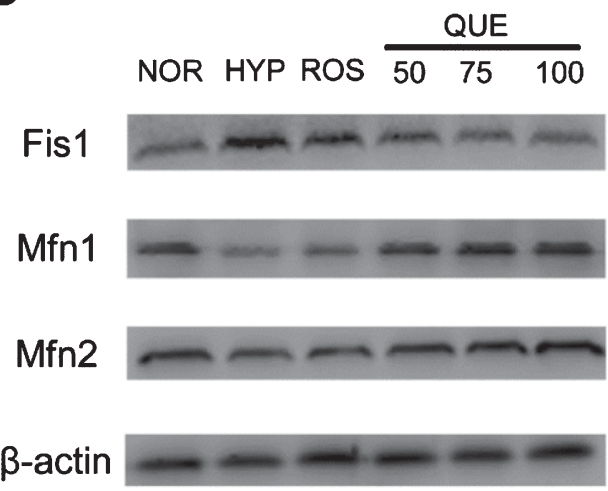

B

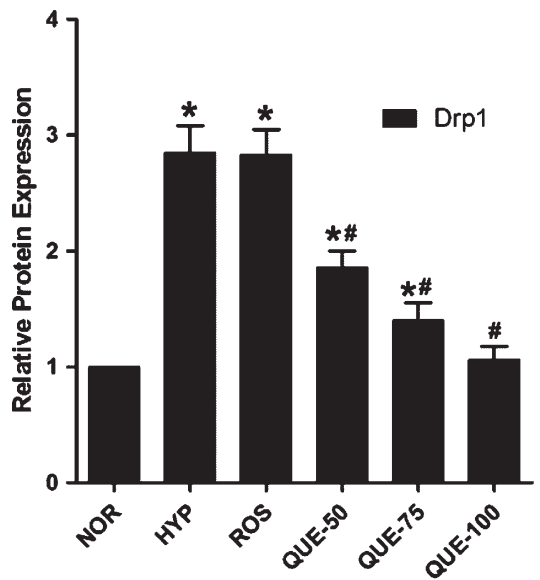

D

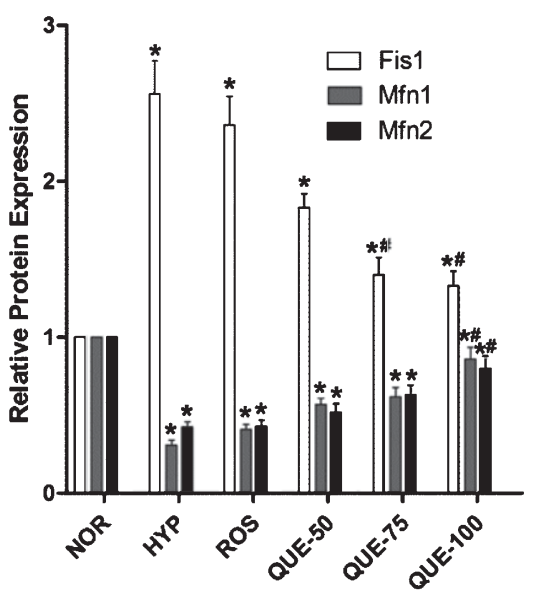

Fig. 7. Quercetin changed the expression of mitochondrial dynamics-related proteins in the hippocampus. (A, C) Representative Western blot images. (B, D) Quantitative analysis showed treatment with quercetin decreased levels of proteins Drp1 and Fis1 significantly and increased levels of proteins Mfn1 and Mfn2 significantly compared to group HYP. ${ }^{*} p<0.05 v s$. group NOR and ${ }^{\#} p<0.05 v s$. group HYP.

that quercetin $(50 \mathrm{mg} / \mathrm{kg} \cdot \mathrm{bw})$ improved memory function in rat exposed to simulated $7600 \mathrm{~m}$ high altitude (Prasad et al., 2013), and indicated that the protective effect was in a concentration-dependent manner with higher doses being ineffective or toxical. Inconsistent with Prasad's reports, we found that the effects of quercetin could not be detected until the dosage reached $50 \mathrm{mg} / \mathrm{kg} \cdot \mathrm{bw}$ in our preliminary tests, but as for the higher dosages including 150 and $200 \mathrm{mg} / \mathrm{kg} \cdot \mathrm{bw}$, the effects of quercetin did not differ significantly from the effect of $100 \mathrm{mg} / \mathrm{kg} \cdot \mathrm{bw}$ assessed by Morris water maze test (data not shown) and no obvious side effect was observed. Owning to the safety and beneficial pharmacological activities, quercetin is a prospective object for food supplementation. In the present study, roseroot, as the common preventive medication for high altitude sickness, was used as an experimental control. Although some scientists have demonstrated its positive effects on cognitive deficit, under our experimental condition, it did not exhibit obvious protective effect against $\mathrm{HH}$-induced memory impairment. Thus, further investigation on the improvement of roseroot on cognitive deficits should be done.

\subsection{Quercetin protects mitochondrial biogenesis, dynamics and function}

Mitochondria, the principal source of energy in the cell, convert nutrients into energy through cellular respiration (Gasser et al., 1982). This 
A

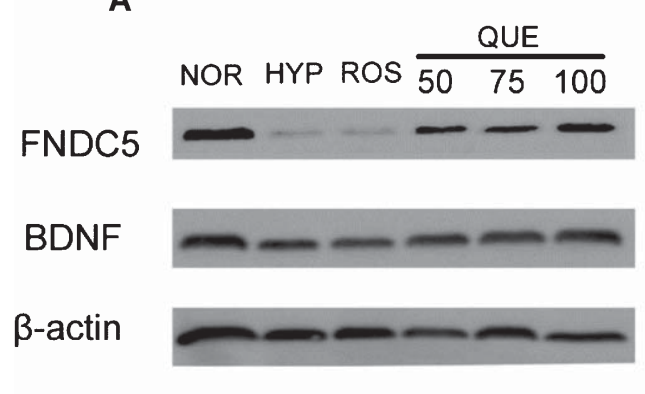

B

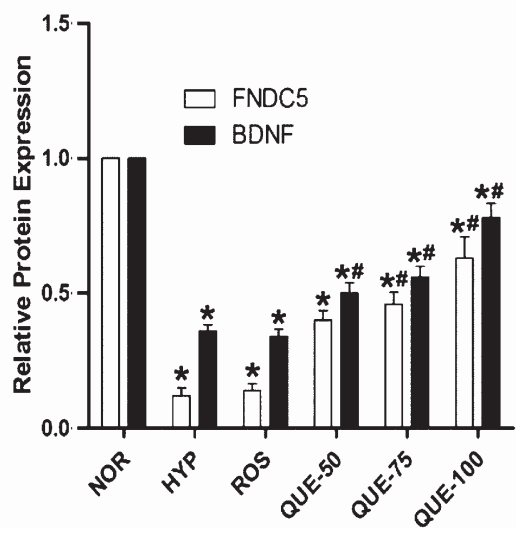

Fig. 8. Quercetin changed the expression of FNDC5 and BDNF in the hippocampus. (A) Representative Western blot images. (B) Quantitative analysis showed treatment with quercetin increased levels of proteins FNDC5 and BDNF significantly compared to group HYP. ${ }^{*} p<0.05 v s$. group NOR and ${ }^{\#} p<0.05$ vs. group HYP.

study identified mitochondrial injury in the hippocampus under $\mathrm{HH}$. This study showed that quercetin was protective of mitochondrial structure and mtDNA copy number, revealing quercetin influenced mitochondrial structure, function and biogenesis. Earlier studies attempted to explain the protective effect of quercetin through its profound antioxidant activity (Sarkar et al., 2012). Recently, however, increasing evidences have emerged to support certain polyphenols could exert numerous ROS-scavenging independent actions. Those studies revealed green tea polyphenols, red wine polyphenols and a red grape polyphenol extract could enhance mitochondrial capacity and biogenesis via NADPH oxidase and nitric oxide synthase pathways (Laurent et al., 2012; Duluc et al., 2013; Rehman et al., 2013). Herein, we suggest PGC- $1 \alpha$, as a regulatory factor of energy metabolism and mitochondrial biogenesis, is involved in the mechanism of neuroprotection by quercetin.

PGC- $1 \alpha$ has a central role in mitochondrial biogenesis and function (Ventura-Clapier et al., 2008). Altering the activity of PGC- $1 \alpha$ induces molecular adaptations that equip the cell to meet the energy demands of a changing environment (Finck and Kelly, 2006). PGC- $1 \alpha$ activity is highly regulated by numerous posttranslational modifications, including phosphorylation and deacetylation (Puigserver et al., 2001; Jaeger et al., 2007; Canto et al., 2009). Sirt1, a type III protein deacetylase, is one of the key upstream kinases for PGC-1 $\alpha$ regulation (Nemoto et al., 2005). Generally, Sirt1 interacts physically with and deacetylates PGC-
$1 \alpha$ at multiple lysine sites and consequently increases PGC- $1 \alpha$ activity (Lagouge et al., 2006). A variety of dietary polyphenols, including resveratrol and quercetin, regulate Sirt1 activity in diabetic and cardiac hypertrophy syndromes (Chung et al., 2010). Data from this study suggested quercetin increased expression of PGC-1 $\alpha$ and Sirt1 in the hippocampus after exposure to $\mathrm{HH}$. Moreover, higher ectopic expression of PGC- $1 \alpha$ (nuclear translocation) in quercetin-treated groups hinted the role of quercetin in activating the Sirt1/PGC- $1 \alpha$ pathway.

Generally, PGC- $1 \alpha$ acts as a transcriptional coactivator through recruitment and co-regulation of multiple transcription factors regulating mitochondrial biogenesis and mtDNA expression (Wu et al., 1999), including Nrf1, Nrf2 and Tfam (Lin et al., 2005). Nrf1 is a $68 \mathrm{kDa}$ transcription factor known to regulate mitochondrial function and biogenesis by integrating nuclear and mitochondrial activities (Kelly and Scarpulla, 2004). The target genes of Nrf1 encode proteins that participate in oxidative phosphorylation, transcription and replication of the mtDNA (Kelly and Scarpulla, 2004). Another well identified transcriptional regulator of cellular energy metabolism is Tfam, which has been shown to initiate a process enabling the catalytic subunit of the mitochondrialspecific polymerase, Polymerase Gamma A, to copy the mtDNA potent regulator of mitochondrial function (Ekstrand et al., 2004). This study suggested quercetin increased expression of Nrf1 and Tfam in rats under $\mathrm{HH}$, which are closely linked to an increase 
in mitochondrial biogenesis. Furthermore, an increase in mtDNA and the number of mitochondria per neuron of rats treated with quercetin suggested the effect on mitochondrial biogenesis. In addition, activity of oxidative respiratory chain complexes were detected, and we found only complex II, complex IV and complex $\mathrm{V}$ activity was enhanced following administration of quercetin after exposure to $\mathrm{HH}$, revealing the protective effect of quercetin on mitochondrial function impaired by HH. ATP levels was also measured, which was in accordance with alterations of respiratory chain complexes activity, providing a further evidence for the protective effect of quercetin on mitochondrial function.

Mitochondria, highly dynamic organelles, continuously undergo the two opposite processes of fission and fusion (Bereiter-Hahn, 1990). Mitochondrial dynamics influence mitochondrial morphology, biogenesis and sub-cellular localization and distribution as well as cell bioenergetics, injury and death (Chan, 2006). Earlier studies highlighted abnormal mitochondrial dynamics relevant to neuronal synaptic loss and cell death in neurodegenerative diseases, including Alzheimer's, Parkinson's and Huntington's diseases (Cho et al., 2010). Exposure to $\mathrm{HH}$ induced mitochondrial dysfunction as described in many reports, but changes of mitochondrial dynamics remain unknown. In the present study, supplementation of quercetin reversed $\mathrm{HH}$-induced decreased mitochondrial surface area and number, revealing that quercetin could improve mitochondrial dynamics during exposure to HH. Drp1 and Fis1 mediate mitochondrial fission. Mfn1, Mfn2 and Opa1 control mitochondrial fusion (Ventura-Clapier et al., 2008). Expression of Mfn $1 / 2$ was increased significantly by treatment with quercetin compared to the $\mathrm{HH}$ group, suggesting quercetin improved the lower level of mitochondrial fusion caused by $\mathrm{HH}$. By contrast, expression of the fission-related proteins Fis 1 and Drp1 was decreased markedly by treatment with quercetin, indicating quercetin could attenuate the elevated mitochondrial fission induced by $\mathrm{HH}$. Considering that fusion/fission processes are an integral part of mitochondrial biogenesis, an alternative explanation for increased mitochondrial biogenesis following treatment with quercetin is the inhibition of mitochondrial degradation owing to decreased fission and increased fusion. These results extended existing findings on the beneficial effects of quercetin on mitochondrial function and biogenesis.

\subsection{Quercetin regulates synaptic plasticity via the PGC-1 $\alpha / F N D C 5 / B D N F$ pathway}

Earlier, it was thought the deleterious effects of exposure to $\mathrm{HH}$ on the hippocampus were reversible and could be negated by returning to sea level for 7-15 days. Recent studies showed cognitive decline at high altitude was associated with sustained neuronal damage and persisted for a long time (Cavaletti et al., 1990; Garrido et al., 1993; Maiti et al., 2007). Generally, dendritic atrophy, mushroom-type spine loss and neuronal apoptosis have been implicated in $\mathrm{HH}-$ induced cognitive deficit (Kauser et al., 2013; Prasad et al., 2013). This study found HH induced the thinner postsynaptic density, impaired mitochondria of hippocampus neurons and was associated with the decreased function of hippocampus neurons, which could be restored by treatment with quercetin. Similar neuroprotective effects of quercetin have also been demonstrated in cerebral ischemia and other oxidative stress-induced neurodegeneration (Heo and Lee, 2004; $\mathrm{Pu}$ et al., 2007). However, besides its anti-oxidative and anti-apoptotic properties, the underlying mechanism of neuroprotective effects of quercetin has not been demonstrated well.

As well as its metabolic regulatory effects, PGC- $1 \alpha$ has important roles in neuron function as evidenced by involvement in the formation and maintenance of neuronal dendritic spines (Cheng et al., 2012). Moreover, Spiegelman (2013) showed this neuroprotective effect was correlated directly with PGC- $1 \alpha$-induced elevation of BDNF expression via FNDC5 (Wrann et al., 2013). BDNF is an important neurotrophin/growth factor. Generally, BDNF is closely related to synaptic plasticity, hippocampal function and learning (Kuipers and Bramham, 2006; Cekic et al., 2012; Paulzen et al., 2014). FNDC5 is identified primarily as a PGC$1 \alpha$-dependent myokine, which is cleaved and secreted from muscle during exercise and induces some major metabolic benefits of exercise (Bostroem et al., 2012). Interestingly, FNDC5 is expressed also in the brain (Dun et al., 2013) and has an important role in neuron development (Hashemi et al., 2013). In this study, we found quercetin treatment promoted the expression of BDNF and FNDC5 in rats compared to untreated rats after acute exposure to $\mathrm{HH}$, which was coincident with quercetin-induced enhanced PGC- $1 \alpha$ expression in the hippocampus. Moreover, the significantly higher levels of BDNF and FNDC5 in quercetin-treated rats highlighted the neuron functional maintenance 
effects of quercetin, which were supported by the relatively integrated synapse observed with TEM and the better performancein Morris water maze test. Previously, Tchantchou et al. (2009) reported that quercetin improved synaptogenesis as well as neurogenesis in hippocampal regions of the brain in a cyclic-AMP Response Element Binding Protein (CREB) dependent manner (Tchantchou et al., 2009). Apart from Sirt1-mediated direct activation of PGC- $1 \alpha$, the transcription of PGC- $1 \alpha$ could be modulated by CREB and AMPK (Palacios et al., 2009). Our results were somehow in a close correspondence with that study. To our knowledge, this is the first evidence of the neuro-protective effect of quercetin through the PGC$1 \alpha /$ FNDC5/BDNF pathway.

In summary, short-term administration of relatively high doses of quercetin can enhance mitochondrial biogenesis, regulate mitochondrial dynamics and increase BDNF expression in the hippocampus in association with reduction of memory impairment following exposure to $\mathrm{HH}$. The prophylactic implications of this are obvious; the data suggest that quercetin might be developed as a drug capable of giving neuro-protection against the deleterious effects of exposure to $\mathrm{HH}$ or improved cognition in related diseases.

\section{Acknowledgments}

This work was funded by research grants from the key projects of the "Twelfth Five-Year Plan" for Medical Science Development of PLA, China (BWS12J034).

\section{Conflicts of interest}

The authors have no conflicts of interest to declare.

\section{References}

Aquilano, K., Vigilanza, P., Baldelli, S., Pagliei, B., Rotilio, G., \& Ciriolo, M.R. (2010). Peroxisome Proliferator-activated Receptor gamma Co-activator 1 alpha (PGC-1 alpha) and Sirtuin 1 (SIRT1) Reside in Mitochondria possible direct function in mitochondrial biogenesis. Journal of Biological Chemistry, 285, 21590-21599.

Bereiter-Hahn, J. (1990). Behavior of mitochondria in the living cell. International Review of Cytology, 122, 1-63.
Bhutada, P., Mundhada, Y., Bansod, K., Bhutada, C., Tawari, S., Dixit, P., \& Mundhada, D. (2010). Ameliorative effect of quercetin on memory dysfunction in streptozotocin-induced diabetic rats. Neurobiology of Learning and Memory, 94(3), 293-302.

Bostroem, P., Wu, J., Jedrychowski, M.P., Korde, A., Ye, L., Lo, J.C., Rasbach, K.A., Bostroem, E.A., Choi, J.H., Long, J.Z., Kajimura, S., Zingaretti, M.C., Vind, B.F., Tu, H., Cinti, S., Hojlund, K., Gygi, S.P., \& Spiegelman, B.M. (2012). A PGC1-alpha-dependent myokine that drives brown-fat-like development of white fat and thermogenesis. Nature, 481, 463$\mathrm{U} 472$.

Canto, C., Gerhart-Hines, Z., Feige, J.N., Lagouge, M., Noriega, L., Milne, J.C., Elliott, P.J., Puigserverand, P., \& Auwerx, J. (2009). AMPK regulates energy expenditure by modulating $\mathrm{NAD}(+)$ metabolism and SIRT1 activity. Nature, 458, 1056-U1140.

Cavaletti, G., Garavaglia, P., Arrigoni, G., \& Tredici, G. (1990). Persistent memory impairment after high altitude climbing. International Journal of Sports Medicine, 11, 176-178.

Cavaletti, G., \& Tredici, G. (1993). Long-lasting neuropsychological changes after a single high altitude climb. Actaneurologica Scandinavica, 87, 103-105.

Cekic, M., Johnson, S.J., Bhatt, V.H., \& Stein, D.G. (2012). Progesterone treatment alters neurotrophin/proneurotrophin balance and receptor expression in rats with traumatic brain injury. Restorative Neurology and Neuroscience, 30(2), 115-126.

Chan, D.C. (2006). Mitochondria: Dynamic organelles in disease, aging, and development. Cell, 125, 1241-1252.

Chan, D.C. (2006). Mitochondrial fusion and fission in mammals. Annual Review of Cell and Developmental Biology, 22, 79-99.

Cheng, A., Wan, R., Yang, J.-L., Kamimura, N., Son, T.G., Ouyang, X., Luo, Y., Okun, E., \& Mattson, M.P. (2012). Involvement of PGC-1 alpha in the formation and maintenance of neuronal dendritic spines. Nature Communications, 3, 1250.

Cho, D.-H., Nakamura, T., \& Lipton, S.A. (2010). Mitochondrial dynamics in cell death and neurodegeneration. Cellular and Molecular Life Sciences, 67, 3435-3447.

Chung, S., Yao, H., Caito, S., Hwang, J.-W., Arunachalam, G., \& Rahman, I. (2010). Regulation of SIRT1 in cellular functions: Role of polyphenols. Archives of Biochemistry and Biophysics, 501, 79-90.

Das, N.P., \& Ratty, A.K. (1986). Effects of flavonoids on induced non-enzymic lipid peroxidation. Progress in Clinical and Biological Research, 213, 243-247.

Davis, J.M., Murphy, E.A., Carmichael, M.D., \& Davis, B. (2009). Quercetin increases brain and muscle mitochondrial biogenesis and exercise tolerance. American Journal of PhysiologyRegulatory Integrative and Comparative Physiology, 296, R1071-R1077.

Duarte, J., Perez Vizcaino, F., Utrilla, P., Jimenez, J., Tamargo, J., \& Zarzuelo, A. (1993). Vasodilatory effects of flavonoids in rat aortic smooth muscle. Structure-activity relationships. General Pharmacology, 24(4), 857-862.

Duluc, L., Jacques, C., Soleti, R., Iacobazzi, F., Simard, G., \& Andriantsitohaina, R. (2013). Modulation of mitochondrial capacity and angiogenesis by red wine polyphenols via estrogen receptor, NADPH oxidase and nitric oxide synthase pathways. 
International Journal of Biochemistry \& Cell Biology, 45(4), 783-791.

Dun, S.L., Lyu, R.M., Chen, Y.H., Chang, J.K., Luo, J.J., \& Dun, N.J. (2013). Irisin-immunoreactivity in neural and non-neural cells of the rodent. Neuroscience, 240, 155-162.

Ekstrand, M.I., Falkenberg, M., Rantanen, A., Park, C.B., Gaspari, M., Hultenby, K., Rustin, P., Gustafsson, C.M., \& Larsson, N.G. (2004). Mitochondrial transcription factor A regulates mtDNA copy number in mammals. Human Molecular Genetics, 13, 935-944.

Finck, B.N., \& Kelly, D.P. (2006). PGC-1 coactivators: Inducible regulators of energy metabolism in health and disease. Journal of Clinical Investigation, 116, 615-622.

Garrido, E., Castello, A., Ventura, J.L., Capdevila, A., \& Rodriguez, F.A. (1993). Cortical atrophy and other brain magnetic resonance imaging (MRI) changes after extremely high-altitude climbs without oxygen. International Journal of Sports Medicine, 14, 232-234.

Gasser, S.M., Daum, G., \& Schatz, G. (1982). Import of proteins into mitochondria. Energy-dependent uptake of precursors by isolated mitochondria. The Journal of Biological Chemistry, 257, 13034-13041.

Hackett, A.M. (1986). The metabolism of flavonoid compounds in mammals. Progress in Clinical and Biological Research, 213 177-194.

Hanasaki, Y., Ogawa, S., \& Fukui, S. (1994). The correlation between active oxygens scavenging and antioxidative effects of flavonoids. Free Radical Biology \& Medicine, 16(6), 845-850.

Hashemi, M.S., Ghaedi, K., Salamian, A., Karbalaie, K., EmadiBaygi, M., Tanhaei, S., Nasr-Esfahani, M.H., \& Baharvand, H. (2013). Fndc5 knockdown significantly decreased neural differentiation rate of mouse embryonic stem cells. Neuroscience, 231, 296-304.

Heo, H.J., \& Lee, C.Y. (2004). Protective effects of quercetin and vitamin $\mathrm{C}$ against oxidative stress-induced neurodegeneration. Journal of Agricultural and Food Chemistry, 52(25), 75147517.

Jaeger, S., Handschin, C., St-Pierre, J., \& Spiegelman, B.M. (2007). AMP-activated protein kinase (AMPK) action in skeletal muscle via direct phosphorylation of PGC-1 alpha. Proceedings of the National Academy of Sciences of the United States of America, 104, 12017-12022.

Jobe, J.B., Shukitt-Hale, B., Banderet, L.E., \& Rock, P.B. (1991). Effects of dexamethasone and high terrestrial altitude on cognitive performance and affect. Aviation, Space, and Environmental Medicine, 62, 727-732.

Kauser, H., Sahu, S., Kumar, S., \& Panjwani, U. (2013). Guanfacine is an effective countermeasure for hypobaric hypdxia-induced cognitive decline. Neuroscience, 254, 110-119.

Kelly, D.P., \& Scarpulla, R.C. (2004). Transcriptional regulatory circuits controlling mitochondrial biogenesis and function. Genes \& Development, 18, 357-368

Kramer, A.F., Coyne J.T., \& Strayer, D.L. (1993). Cognitive function at high altitude. Human Factors, 35, 329-344.

Kuipers, S.D., \& Bramham, C.R. (2006). Brain-derived neurotrophic factor mechanisms and function in adult synaptic plasticity:
New insights and implications for therapy. Current Opinion in Drug Discovery \& Development, 9, 580-586.

Kumar, A., Sehgal, N., Kumar, P., Padiand, S.S.V., \& Naidu, P.S (2008). Protective effect of quercetin against ICV colchicineinduced cognitive dysfunctions and oxidative damage in rats. Phytotherapy Research, 22(12), 1563-1569.

Lagouge, M., Argmann, C., Gerhart-Hines, Z., Meziane, H., Lerin, C., Daussin, F., Messadeq, N., Milne, J., Lambert, P., Elliott, P. Geny, B., Laakso, M., Puigserver, P., \& Auwerx, J. (2006). Resveratrol improves mitochondrial function and protects against metabolic disease by activating SIRT1 and PGC-1 alpha. Cell, 127, 1109-1122.

Laurent, C., Chabi, B., Fouret, G., Py, G., Sairafi, B., Elong, C., Gaillet, S., Cristol, J.P., Coudray, C., \& Feillet-Coudray, C. (2012). Polyphenols decreased liver NADPH oxidase activity, increased muscle mitochondrial biogenesis and decreased gastrocnemius age-dependent autophagy in aged rats. Free Radical Research, 46(9), 1140-1149.

Lin, J.D., Handschinand, C., \& Spiegelman, B.M. (2005). Metabolic control through the PGC-1 family of transcription coactivators. Cell Metabolism, 1, 361-370.

Magalhaes, J., Ascensao, A., Soares, J.M.C., Ferreira, R., Neuparth, M.J., Marques, F., \& Duarte, J.A. (2005). Acute and severe hypobaric hypoxia increases oxidative stress and impairs mitochondrial function in mouse skeletal muscle. Journal of Applied Physiology, 99, 1247-1253.

Maiti, P., Muthuraju, S., Ilavazhagan, G., \& Singh, S.B. (2008) Hypobaric hypoxia induces dendritic plasticity in cortical and hippocampal pyramidal neurons in rat brain. Behavioural Brain Research, 189, 233-243.

Maiti, P., Singh, S.B., Muthuraju, S., Veleri, S., \& Ilavazhagan, G. (2007). Hypobaric hypoxia damages the hippocampal pyramidal neurons in the rat brain. Brain Research, 1175, 1-9.

Morris, R. (1984). Developments of a water-maze procedure for studying spatial learning in the rat. Journal of Neuroscience Methods, 11, 47-60.

Morris, R.G., Garrud, P., Rawlins, J.N., \& O'Keefe, J. (1982). Place navigation impaired in rats with hippocampal lesions. Nature, 297, 681-683.

Muthuraju, S., Maiti, P., Solanki, P., Sharma, A.K., Amitabh, S.B. Singh, D.P., \& Ilavazhagan, G. (2009). Acetylcholinesterase inhibitors enhance cognitive functions in rats following hypobaric hypoxia. Behavioural Brain Research, 203, $1-14$.

Nemoto, S., Fergusson, M.M., \& Finkel, T. (2005). SIRT1 functionally interacts with the metabolic regulator and transcriptional coactivator PGC-1 alpha. Journal of Biological Chemistry, 280, 16456-16460.

Palacios, O.M., Carmona, J.J., Michan, S., Chen, K.Y., Manabe, Y., Ward, J.L., Goodyear, L.J., \& Tong, Q. (2009). Diet and exercise signals regulate SIRT3 and activate AMPK and PGC-1 alpha in skeletal muscle. Aging-Us, 1(9), 771-783.

Paulzen, M., Veselinovic, T., \& Gruender, G. (2014). Effects of psychotropic drugs on brain plasticity in humans. Restorative Neurology and Neuroscience, 32(1), 163-181.

Prasad, J., Baitharu, I., Sharma, A.K., Dutta, R., Prasad, D., \& Singh, S.B. (2013). Quercetin reverses hypobaric hypoxia-induced 
hippocampal neurodegeneration and improves memory function in the rat. High Altitude Medicine \& Biology, 14(4), 383-394.

Pu, F., Mishima, K., Irie, K., Motohashi, K., Tanaka, Y., Orito, K., Egawa, T., Kitamura, Y., Egashira, N., Iwasaki, K., \& Fujiwara, M. (2007). Neuroprotective effects of quercetin and rutin on spatial memory impairment in an 8-arm radial maze task and neuronal death induced by repeated cerebral ischemia in rats. Journal of Pharmacological Sciences, 104(4), 329-334.

Pu, F.L., Mishima, K., Egashira, N., Iwasaki, K., Kaneko, T., Uchida, T., Irie, K., Ishibashi, D., Fujii, H., Kosuna, K., \& Fujiwara, M. (2004). Protective effect of buckwheat polyphenols against long-lasting impairment of spatial memory associated with hippocampal neuronal damage in rats subjected to repeated cerebral ischemia. Journal of Pharmacological Sciences, 94, 393-402.

Puigserver, P., Rhee, J., Lin, J., Wu, Z., Yoon, J.C., Zhang, C.Y., Krauss, S., Mootha, V.K., Lowell, B.B., \& Spiegelman, B.M. (2001). Cytokine stimulation of energy expenditure through p38 MAP kinase activation of PPARgamma coactivator-1. Molecular Cell, 8, 971-982.

Rehman, H., Krishnasamy, Y., Haque, K., Thurman, R.G., Lemasters, J.J., Schnellmann, R.G., \& Zhong, Z. (2013). Green tea polyphenols stimulate mitochondrial biogenesis and improve renal function after chronic cyclosporin a treatment in Rats. Plos One, 8(6), e65029.

Sarkar, A., Angeline, M.S., Anand, K., Ambasta, R.K., \& Kumar, P. (2012). Naringenin and quercetin reverse the effect of hypobaric hypoxia and elicit neuroprotective response in the murine model. Brain Research, 1481, 59-70.

Shukitt-Hale, B., Banderet, L.E., \& Lieberman, H.R. (1998). Elevation-dependent symptom, mood, and performance changes produced by exposure to hypobaric hypoxia. Int $J$ AviatPsychol, 8, 319-334.

Shukitt-Hale, B., Kadar, T., Marlowe, B.E., Stillman, M.J., Galli, R.L., Levy, A., Devine, J.A., \& Lieberman, H.R. (1996). Mor- phological alterations in the hippocampus following hypobaric hypoxia. Human \& Experimental Toxicology, 15, 312-319.

Silva, B., Oliveira, P.J., Dias, A., \& Malva, J.O. (2008). Quercetin, kaempferol and biapigenin from Hypericumperforatum are neuroprotective against excitotoxic insults. Neurotoxicity Research, 13, 265-279.

Sriraksa, N., Wattanathorn, J., Muchimapura, S., Tiamkao, S., Brown, K., \& Chaisiwamongkol, K. (2012). Cognitiveenhancing effect of quercetin in a rat model of Parkinson's disease induced by 6-hydroxydopamine. Evidence-based complementary and alternative medicine: eCAM, 2012, 823206-823206.

Tchantchou, F., Lacor, P.N., Cao, Z., Lao, L., Hou, Y., Cui, C., Klein, W.L., \& Luo, Y. (2009). Stimulation of neurogenesis and synaptogenesis by bilobalide and quercetin via common final pathway in hippocampal neurons. Journal of Alzheimers Disease, 18(4), 787-798.

Ventura-Clapier, R., Garnier, A., \& Veksler, V. (2008). Transcriptional control of mitochondrial biogenesis: The central role of PGC-1 alpha. Cardiovascular Research, 79, 208-217.

Wang, J., Ke, T., Zhang, X., Chen, Y., Liu, M., Chen, J., \& Luo, W. (2013). Effects of acetazolamide on cognitive performance during high-altitude exposure. Neurotoxicology and Teratology, 35, 28-33.

Weibel, E.R. (1979). Morphometry of the human lung: The state of the art after two decades. Bulletin Europeen de Physiopathologie Respiratoire, 15(5), 999-1013.

Wrann, C.D., White, J.P., Salogiannnis, J., Laznik-Bogoslavski, D., Wu, J., Ma, D., Lin, J.D., Greenberg, M.E., \& Spiegelman, B.M. (2013). Exercise Induces Hippocampal BDNF through a PGC-1 alpha/FNDC5 Pathway. Cell Metabolism, 18, 649-659.

Wu, Z., Puigserver, P., Andersson, U., Zhang, C., Adelmant, G., Mootha, V., Troy, A., Cinti, S., Lowell, B., Scarpulla, R.C., \& Spiegelman, B.M. (1999). Mechanisms controlling mitochondrial biogenesis and respiration through the thermogeniccoactivator PGC-1. Cell, 98, 115-124. 